Voix et Images

voixetimages

\title{
Bibliographie de Claire Martin
}

\section{Gilles Dorion}

Volume 29, numéro 1 (85), automne 2003

\section{Claire Martin}

URI : https://id.erudit.org/iderudit/007541ar

DOI : https://doi.org/10.7202/007541ar

Aller au sommaire du numéro

\section{Éditeur(s)}

Université du Québec à Montréal

\section{ISSN}

0318-9201 (imprimé)

1705-933X (numérique)

Découvrir la revue

Citer ce document

Dorion, G. (2003). Bibliographie de Claire Martin. Voix et Images, 29(1), 87-101.

https://doi.org/10.7202/007541ar d'utilisation que vous pouvez consulter en ligne.

https://apropos.erudit.org/fr/usagers/politique-dutilisation/ 


\section{B I B L I O G R A P H I E \\ de Claire Martin}

$+++$

\section{GILLES DORION}

Université Laval

\section{E U V R E S}

\section{1. Nouvelles (en volumes)}

+ Avec ou sans amour, Montréal, Le Cercle du livre de France, 1958, 186 p.; Avec ou sans amour. Nouvelles, Paris, Robert Laffont, 1959, 205 p. ; Avec ou sans amour, présentation et annotations de Robert Vigneault, Montréal, Éditions du Renouveau pédagogique inc., coll. «Lecture Québec», 1969, 187 p.; Montréal, Le Cercle du livre de France, coll. «CLF poche», n²7, 1970, 157 p.; Avec ou sans amour. Nouvelles, [I. «Rupture» et II «Faux Départ»], 2 vol., Hull, Éditions E.L.V.O., 1985, 142 p.; Love Me, Love Me Not, trad. David Lobdell, Ottawa, Oberon Press, 1987, 142 p.

+ Toute la vie, présentation de Gilles Dorion, Québec, L'instant même, 1999, 113 p.

\section{2. Ro m a n s}

+ Doux-amer. Roman, Montréal, Le Cercle du livre de France, 1960, 192 p.; Paris, Robert Laffont, 1960, 222 p.; Montréal, Le Cercle du livre de France, coll. «CLF poche», n 3, 1967, 166 p.; présentation et bibliographie par Aurélien Boivin, Montréal, Bibliothèque québécoise, 1999, 212 p.; Best Man, trad. David Lobdell, Ottawa, Oberon Press, 1983, 149 p.

+ Quand j'aurai payé ton visage. Roman, Montréal, Le Cercle du livre de France, 1962, 187 p. ; Paris, Robert Laffont, 1962, 187 p.; The Legacy, trad. David Lobdell, Ottawa, Oberon Press, 1986, 149 p.

+ Les morts. Roman, Montréal, Le Cercle du livre de France, 1970, 152 p.

+ L'amour impuni. Roman, Québec, L'instant même, 2000, 145 p.

+ La brigande. Roman, Québec, L'instant même, 2001, 188 p.

+ Il s'appelait Thomas, Québec, L'instant même, 2003, 204 p.

\section{3. M é moires}

+ Dans un gant de fer. Première Partie. La joue gauche, Montréal, Le Cercle du livre de France, 1965, 235 p.; Dans un gant de fer II. La joue droite, Montréal, Le Cercle du livre de France, 1966, 209 p. ; Montréal, Le Cercle du livre de France, 1978, 235 et 209 p. ; Dans un gant de fer. La joue gauche, présentation et bibliographie d'Aurélien Boivin, Montréal, Bibliothèque québécoise, 1999, 230 p.; In an Iron Glove, trad. Philip Stratford, Toronto, The Ryerson Press, 1968, 327 p.; The Right Cheek: An Autobiography by Claire Martin, trad. Philip Stratford, Montréal, Harvest House, coll. «French Writers of Canada», 1975, 161 p. Les pages 131-133 de Dans un gant de fer ont paru dans Liberté, n 26, mars-avril, 1963, p. 131-138; les pages 67-75 de La joue droite ont paru dans Le Devoir, 31 mars 1966, p. 18; les pages 73 et 75 de La joue droite ont été reproduites dans Michel Lebel et Jean-Marcel Paquette, sous le titre «Spectacles de 
couvent», dans Le Québec par ses textes littéraires (1534-1976), Montréal/Paris, Fernand Nathan, 1979, p. 274-276.

\section{I . 4. T h é ât r e}

+ «Moi, je n'étais qu'espoir», Montréal, Le Cercle du livre de France, 1972, 54 p.

\section{5. R é cit}

+ La petite fille lit, Ottawa, Éditions de l'Université d'Ottawa, Publication du Département des lettres françaises, coll. «Textes» n² 2, 1973, 18 p. (repris dans Le choix de Claire Martin dans l'œuvre de Claire Martin et dans Toute la vie).

\section{I.6. Anthologie}

+ Le choix de Claire Martin dans l'œuvre de Claire Martin, Charlesbourg, Les Presses Laurentiennes, coll. «Le choix de...», 1984, 79 p.

\section{7. Traductions}

+ [Patsauq], Markoosie, Le harpon du chasseur. Roman [Harpoon of the Hunter], trad. de l'anglais par Claire Martin, Montréal, Le Cercle du livre de France, 1971, 95 p.

+ Pitseolak, [Peter], Le livre d'images de ma vie [Pictures Out of my Life], tiré des interviews enregistrées par Dorothy Eber, texte français de Claire Martin, Montréal, Le Cercle du livre de France, 1972, [n.p.]

+ Laurence, Margaret, L'ange de pierre [The Stone Angel], trad. de l'anglais par Claire Martin, Montréal, CLF Pierre Tisseyre, coll. «Des deux Solitudes», 1976, 342 p.

+ Allen, Robert Thomas, Le violon [The Violin], trad. de l'anglais par Claire Martin, Montréal, CLF Pierre Tisseyre, coll. «Des deux Solitudes. Juvénile», 1976, 78 p.

+ Davies, Robertson, Le lion avait un visage d'homme. Roman [The Manticore], trad. de l'anglais par Claire Martin, Montréal, CLF Pierre Tisseyre, 1978, 323 p.

+ Davies, Robertson, Le monde des merveilles [World of Wonders], trad. de l'anglais par Claire Martin, Montréal, CLF Pierre Tisseyre, coll. «Des deux Solitudes», 1979, 417 p.

+ Blaise, Clark, La justice tribale. Récits [Tribal Justice], trad. de l'anglais par Claire Martin, Montréal, CLF Pierre Tisseyre, coll. «Des deux Solitudes», 1985, 268 p.

\section{I.8. Articles et récits brefs dans des livres et des périodiques}

+ «Colette», Bulletin. Société d'étude et de conférences, mars 1954, p. 79-84.

+ «De ce que la nécessité est mère de l'invention», Bulletin. Société d'étude et de conférences, février 1956, p. 44-48 (repris dans Avec ou sans amour sous le titre «Nécessité, mère de l'invention»).

+ «Toute la vie», Liberté, n 1, janvier-février 1959, p. 31-32 (repris dans Toute la vie).

+ «Le risque d'être dupe», Liberté, n 4, juillet-août 1959, p. 238-241 (repris dans Toute la vie).

+ «Notre roman, image de notre milieu», Revue dominicaine, juillet-août 1960, p. 18-24.

+ «Le français sans maître au Canada», Le Devoir, «Supplément littéraire», 22 octobre 1960, p. 11.

+ «La nouvelle chanson», Châtelaine, juillet 1961, p. 25, 58 et 60 (repris dans Karch, Mariel O’Neil et Pierre Paul Karch, Options, Toronto, Oxford University Press, 1974, p. 75-82 et dans Toute la vie).

+ «Un homme», Le Magazine Maclean, juillet 1961, p. 25 et 38 (repris dans Toute la vie).

+ «L'homme dans le roman canadien-français», Incidences, avril 1964, p. 5-8.

+ «Témoignages des romanciers canadiens-français», Archives des lettres canadiennes, t. III, Le roman canadienfrançais. Évolution-Témoignages-Bibliographie, Montréal, Fides, 1964, p. 346-352; rééd. 1971 et 1977, p. 383389.

+ «Ā propos de ces nouvelles techniques», Incidences, mai 1965, p. 15-20. 
+ «Ā propos de son autobiographie. Témoignages» Le Devoir, 27 octobre 1966, p. 36.

+ «Three Points in my Life. At Three and a Half», trad. Philip Stratford, The Tamarack Review, printemps 1967, p. 5-18.

+ «Situation de la littérature québécoise. Faut-il trouver des catégories!», Le Devoir, «Supplément littéraire», 31 octobre 1967, p. X.

+ «Une question, des réponses», Liberté, n 54, novembre-décembre 1967, p. 35-36.

+ «Paris-Montréal», Europe, février-mars 1969, p. 77-82 (repris dans Le choix de Claire Martin dans l'œuvre de Claire Martin et dans Toute la vie).

+ «Suis-moi» (Avec ou sans amour) a paru en serbe, dans Smederevo. Revue pour la culture, l'art et les questions sociales, décembre 1987, p. 9-11, Smederevo, Yougoslavie.

+ «Lettre ouverte à un ami lointain et dont je ne sais presque plus rien», Écrits du Canada français, n 81 , 3e trimestre 1994, p. 5-16.

+ «Combien j'ai douce souvenance», Les Écrits, n 84, $3^{e}$ trimestre 1995, p. 47-60 (repris dans Toute la vie).

+ "Que reste-t-il de nos amours?», Gilles Pellerin (dir.), Québec. Des écrivains dans la ville, Québec, L'instant même/Musée du Québec, 1995, p. 81-83.

+ «Un fleuve», Les Écrits, n 90, 3e trimestre 1997, p. 5-12 (repris dans Toute la vie).

+ «Histoire enveloppée», Les Écrits, n 95, 2 trimestre 1999, p. 59-62 (repris dans Toute la vie).

+ «Les fantômes de mon enfance», Châtelaine, juin 2000, p. 68-70.

+ «La première personne du singulier», Les Écrits, n 99, août 2000, p. 61-65.

+ "Je ne suis pas revenue de tout», Gilles Pellerin (dir.), Les travaux de Philocrate Bé découvreur de mots suivis d'une biographie d'icelui, Québec, L'instant même/Musée de la civilisation, 2000, p. 81-93.

+ «Le vert paradis et le chapeau noir», De la couleur des mots 7, regards, histoires, Montréal, Les 400 coups, 2001, p. 15-21.

+ «Pauvre Joyce! Moi non plus!», Nuit blanche, nº 91, été 2003, p. 28-29.

\section{ARTICLES ET COMPTES RENDUS SUR CLAIRE MARTIN ET SON E U V R E}

\section{1. Avec ou sans amour}

+ [ANONYME], «Une femme gagne le prix du Cercle du livre de France», La Presse, 16 octobre 1958, p. 6.

+ — , [Deux photos, avec légende: «Claire Martin est l'heureuse récipiandaire [sic] du “Prix du Cercle du livre de France", pour son livre Avec ou sans amour, qui sera publié bientôt [...]»], Radiomonde et Télémonde, 25 octobre 1958, p. 3.

+ - - "Avec ou sans amour de Claire Martin", L'Action catholique, 15 novembre 1958, p. 4.

+ _- "Avec ou sans amour et L'Anse aux brumes édités», Le Devoir, 15 novembre 1958, p. 4.

+ — - "Claire Martin chez les femmes universitaires», Le Droit, 3 décembre 1958, p. 4.

+ — , «La littérature voyage. Romans canadiens édités à Paris», Le Petit Journal, 29 mars 1959, p. 110.

+ —_ «La sélection des libraires de France», L'Authie, 2 mai 1959.

+ — - "Accueil favorable à un Prix du Cercle», La Presse, 30 janvier 1960, p. 37.

+ BEAUCHEMIN, Lucette, "Ce dont on parle», La Revue populaire, décembre 1958, p. 45.

+ BOUCHER, Jean-Pierre, "Amour et narration. Avec ou sans amour de Claire Martin», Le recueil de nouvelles. Études sur un genre littéraire dit mineur, Montréal, Fides, 1992, p. 131-143.

+ BRAIS, Lucille, "Avec ou sans amour», Bulletin. Société d'étude et de conférences, décembre 1958, p. 33-34.

+ CHAPUT-ROLLAND, Solange, «Les livres. Trois femmes... deux hommes», Points de vue, janvier 1959, p. 18.

+ DALLARD, Sylvie, "Avec ou sans amour, recueil de nouvelles de Claire Martin (pseudonyme de Claire Montreuil)", Maurice Lemire et al., Dictionnaire des œuvres littéraires du Québec, t. 3, 1940-1959, Montréal, Fides, 1982, p. 101-103; rééd. Saint-Laurent, Fides, 1995. 
+ DIONNE, René, "Claire Martin: Avec ou sans amour», Relations, août 1959, p. 221.

+ DUHAMEL, Roger, «Courrier des lettres. Claire Martin s'inscrit parmi les meilleurs conteurs de notre littérature», La Patrie du dimanche, 18 janvier 1959, p. 34.

+ G[ODIN], G[érald], «La vie des Lettres», Le Nouvelliste, 9 janvier 1960, p. 12.

+ GRANDPRÉ, Pierre de, "Couronnées par le Cercle du livre de France. Les nouvelles de Claire Martin», Le Devoir, 13 décembre 1958, p. 11 (repris dans Dix ans de vie littéraire au Canada français, p. 158-161, sous le titre «Comment l'esprit vient aux filles»).

+ HAMELIN, Jean, « $\mathrm{M}^{\mathrm{me}}$ Claire Martin, 10e prix du Cercle du livre de France», Le Devoir, 16 octobre 1958, p. 3.

+ — - «Le prix du Cercle du livre de France. Avec ou sans amour, de Claire Martin», Le Devoir, 6 décembre 1958, p. 9 et 11.

+ HÉNAULT, Gilles, "Claire Martin: "Mes histoires sont souvent des jugements téméraires" », Le Devoir, 20 août 1960, p. 7.

+ LECLERC, Rita, "Notices bibliographiques. Littérature canadienne. Martin (Claire), Avec ou sans amour», Lectures, $1^{\mathrm{er}}$ janvier 1959, p. 134.

+ LEMAR, Yves, «Les écrivains canadiens s'émancipent (enfin)», Arts, 2 décembre 1959, Paris.

+ LOMBARD, Pierre [pseudonyme de Pierre-Paul Turgeon], «Claire... de l'une et de l'autre», L'Action catholique, 13 décembre 1958, p. 4.

+ - _ "Avec ou sans amour», L'Action catholique, 20 décembre 1958, p. 4.

+ LOSIC, Serge, «Avec ou sans amour. L'amour dans l'œuvre de Claire Martin», Le Devoir, 7 novembre 1964, p. 28-29.

+ MARULLO, Claude, "Quand Claire Martin incarne la femme québécoise d'avant 1960», Co-Incidences, marsavril 1974, p. 17-31.

+ PARENT, Darquise, «Hommage à Claire Martin», Le Droit, 5 novembre 1958, p. 7.

+ PILOTTE, Hélène, «Claire Martin, l'auteur de Avec ou sans amour», Le Devoir, 29 novembre 1958, p. 15.

+ PIUZE, Suzanne, «Instantanés féminins. Avec ou sans amour», La Patrie du dimanche, 19 octobre 1958, p. 100.

+ POLIQUIN, Jean-Marc, «La Colette de la région outaouaise», Le Droit, 20 décembre 1958, p. 4.

+ ROBERT, Guy, «Petit bilan de notre littérature 1958», Revue dominicaine, mars 1959, p. 86-87.

+ ROBILLARD, Jean-Paul, "Le livre de la semaine. Avec ou sans amour», Le Petit Journal, semaine du 21 au 28 décembre 1958, p. 31.

+ SAINT-MARTIN, Fernande, «Entrevue avec Claire Martin. Plus qu'un homme, la femme doit attendre la maturité pour écrire», La Presse, 16 octobre 1958, p. 18.

+ VALOIS, Marcel [pseudonyme de Jean Dufresne], «Sans amour», La Presse, 13 décembre 1958, p. 42.

+ VANBRUGGHE, Annick, "De la tentation de la lucidité à l'amour dépoétisé: Avec ou sans amour, de Claire Martin», Livres et Auteurs québécois, 1969, p. 231-236.

+ VIGNEAULT, Robert, «Présentation» (p. 9-24), «Repères biographiques» (p. 25), "Annotations (passim)», «Bibliographie» (p. 185-186) et «Glossaire» (p. 187-188), dans Claire Martin, Avec ou sans amour, édition de 1969.

\section{2. Doux-amer}

+ [ANONYME], «Un livre canadien dans la course pour un grand prix?», Le Devoir, 2 avril 1960, p. 9.

+ + - «Doux-amer, un premier roman de Claire Martin», La Presse, 3 septembre 1960, p. 27.

+ — , «Doux-amer de Claire Martin», Le Nouvelliste, 3 septembre 1960, p. 11.

+ ——, «À Paris. Lancement de Doux-amer», La Presse, 28 septembre 1960, p. 32.

+ + - "Claire Martin: Ottawa vaut bien un livre», Le Figaro, 6 octobre 1960, p. 23.

+ —_, «Doux-amer», Fiches bibliographiques de littérature canadienne, avril 1968.

+ BELLEAU, André, "Chroniques. Les livres», Liberté, n 12, novembre-décembre 1960, p. 376-378.

+ DUHAMEL, Roger, "Le livre de la semaine. Doux-amer», La Patrie du dimanche, section «Magazine», 27 novembre 1960, p. 19. 
+ — , «Roman et thêâtre», University of Toronto Quarterly, juillet 1961, p. 484-486 (repris dans Gilles Marcotte, Présence de la critique, p. 62-63, sous le titre «Un sujet permanent...»).

+ FRANCION [pseudonyme de Lisette Morin], "Auteur canadien ou livre canadien?», Le Progrès du Golfe, 14 octobre 1960, p. 5 et 12.

+ FRENCH, William, "Rural Life, Urban Chic», The Globe and Mail, 25 juin 1983, p. 16.

+ GAY, Paul, «Doux-amer», Le Droit, 22 octobre 1960, p. 11.

+ GODIN, Gérald, «Le libraire et Doux-amer de Gérard Bessette et Claire Martin», Le Nouvelliste, 5 novembre 1960, p. 11.

+ H[AMELIN], J[ean], «Robert Laffont et la diffusion du livre canadien en France», La Presse, 8 octobre 1960, p. 34.

+ HAMELIN, Jean, «La vie littéraire. Deux romans canadiens. Doux-amer, de Claire Martin [...]», La Presse, 22 octobre 1960, p. 36.

+ KATTAN, Naïm, «Les livres. Doux-amer, roman par Claire Martin», Bulletin du Cercle juif, novembre 1960, p. 3.

+ L[ACOMBE], L[ia], «Douceur et amertume de l'amour», Les Lettres françaises, nº 850, 17-23 novembre 1960, p. 3.

+ L[ECLERC], R[ita], «Martin (Claire), Doux-amer», Lectures, novembre 1960, p. 78.

+ LOCKQUELL, Clément, «Doux-amer de Claire Martin. Le roman de l'égoïsme», Le Devoir, 29 octobre 1960, p. 11.

+ MAILHOT, Michèle A., "Quatre excellentes femmes écrivains», Châtelaine, janvier 1961, p. 7.

+ MÉNARD, Jean, «Le roman et le conte», Revue de l'Université d'Ottawa, avril-juin 1961, p. 313-314.

+ OWEN, Patricia, «All Is Forgiven?», The Tamarack Review, n 52, 1969, p. 80-82.

+ PASCAL, Gabrielle, "Doux-amer, roman de Claire Martin [née Montreuil]», Maurice Lemire et al., Dictionnaire des œuvres littéraires du Québec, t. IV, 1960-1969, Montréal, Fides, 1984, p. 275-276.

+ P[IVOT], B[ernard], «Deux éditeurs pour un roman», Le Figaro littéraire, 8 octobre 1960, p. 2.

+ RENAUD, André, "L'héroïne du roman canadien et l'expérience de l'amour», Archives des lettres canadiennes, t. III, Le roman canadien-français. Évolution-Témoignages-Bibliographie, Montréal, Fides, 1964, p. 183-196 (voir p. 189-192).

+ ROBIDOUX, Réjean, «Le roman canadien-français de demain», Archives des lettres canadiennes, t. III, Le roman canadien-français. Évolution-Témoignages-Bibliographie, Montréal, Fides, 1964, p. 241-256 (voir p. 251-252).

+ ROBIDOUX, Réjean et André RENAUD, Le roman canadien-français du vingtième siècle, Ottawa, Éditions de l’Université d'Ottawa, 1966, p. 147-162.

\section{II.3. Quand j'aurai payé ton visage}

+ [ANONYME], «Les livres. Quand j'aurai payé ton visage, par Claire Martin», Bulletin du Cercle juif, juillet 1962, p. 3.

+ ——, "À livre ouvert», La Semaine à Radio-Canada, 25 juillet 1964, p. 16.

+ —_ "Claire Martin et Pauline Julien à "Votre choix"», La Semaine à Radio-Canada, 20 novembre 1965, p. 10.

+ ARCHAMBAULT, Gilles, "Quand j’aurai payé ton visage», Livres et Auteurs canadiens, 1962, p. 26-27.

+ BEAUPARLANT, C. «D'autres ont lu pour nous», Châtelaine, novembre 1962, p. 100.

+ BLAIN, Maurice, «Quand j'aurai payé ton visage, roman de Claire Martin. Comment fille inconstante devient femme fidèle», Le Nouveau Journal (supplément), 19 mai 1962, p. iv (repris dans Gilles Marcotte, Présence de la critique, p. 167-169).

+ CHANTAL, Geneviève de, "Actualités littéraires. Les romancières et l'amour», Le Droit, 26 mai 1962, p. 9.

+ DORION, Gilles, "Quand j'aurai payé ton visage, roman de Claire Martin (née Montreuil)», Maurice Lemire et al., Dictionnaire des œuvres littéraires du Québec, t. IV, 1960-1969, Montréal, Fides, 1984, p. 744-746. 
+ DUHAMEL, Roger, «Le livre de la semaine. Quand j'aurai payé ton visage», La Patrie du dimanche, 6 mai 1962, p. 17 (repris dans Gilles Marcotte, Présence de la critique, p. 65-68, sous le titre «La maîtrise d'une écriture»).

+ ÉTHIER-BLAIS, Jean, «Romans et théâtre», University of Toronto Quarterly, juillet 1963, p. 500, 502-503.

+ — - «La mère et l'eau», Le Devoir, 6 juin 1964, p. 13 (repris dans Signets II, p. 224-228).

+ FOREST, Gilbert, «Quelques propos sur la littérature canadienne. IV. Le roman», Collège et Famille, février 1964, p. 22-23.

+ GAY, Paul, «Quand j'aurai payé ton visage», Le Droit, 5 mai 1962, p. 6.

+ HAMELIN, Jean, «Quand j'aurai payé ton visage, de Claire Martin», Le Devoir, 28 avril 1962, p. 13.

+ KEABLE, Jacques, «Troisième livre de Claire Martin: Quand j'aurai payé ton visage», La Presse, 18 avril 1962 , p. 49.

+ LECLERC, Rita, «Notices bibliographiques. Littérature canadienne. Martin (Claire), Quand j'aurai payé ton visage», Lectures, juin 1962, p. 283-284.

+ MAILHOT, Michèle A., "Pour qui aime hommes et bêtes», Châtelaine, septembre 1962, p. 48.

+ MARCOTTE, Gilles, «Claire Martin et la théorie de l'amour», La Presse (supplément), 28 avril 1962, p. 9.

+ MÉNARD, Jean, «Le dernier roman de Claire Martin», Incidences, novembre 1962, p. 38-41 (repris dans La vie littéraire au Canada français, p. 216-219).

+ ROBERT, Guy, «Une demi-douzaine de chefs-d’œuvre s.v.p. La romancière Claire Martin», Maintenant, juin 1962, p. 230-231.

+ ROBIDOUX, Réjean, "Le roman canadien-français de demain», Archives des lettres canadiennes, t. III, Le roman canadien-français. Évolution-Témoignages-Bibliographie, Montréal, Fides, 1964, p. 241-265 (voir p. 250-251).

+ ROBIDOUX, Réjean et André RENAUD, Le roman canadien-français du vingtième siècle, Ottawa, Éditions de l'Université d'Ottawa, 1966, p. 150-154.

+ SAINT-ONGE, Paule, «The French-Canadian Novel-1962», The Canadian Author and Bookman, printemps 1963, p. 4.

+ SANGLOT, Arthur, "Au Canada du nouveau. La psychologie l'emporte sur la couleur locale», La Tribune de Genève, 20 janvier 1963.

+ TISSEYRE, Pierre, «À livre ouvert», La Revue populaire, mai 1962, p. 12.

\section{II.4. Dans un gant de fer et La joue droite}

+ [ANONYME], «Châtelaine en pantoufles», Châtelaine, juin 1965, p. 3.

+- , «En quelques mots [...]. Dans un gant de fer», Le Bien public, 14 janvier 1966, p. 6.

+ — , «Aquin édité à Paris et Martin rééditée à Montréal», La Presse (supplément), 26 février 1966, p. 2.

+ + — «M.-C. Blais et C. Martin: Prix France-Québec 1966», Le Devoir, 2 avril 1966, p. 11.

$+\ldots$, «Parmi les lauréats des Concours de la province, des noms familiers aux Québécois», Le Soleil, 22 octobre 1966, p. 10.

+ — - «Votre première sélection. La joue droite de Claire Martin», Le Soleil, 22 octobre 1966, p. 11 [publicité du CLF].

+ ——, «La joue droite», Fiches bibliographiques de littérature canadienne, novembre 1966.

+ — , «Prix littéraires du Gouverneur général», Le Devoir, 25 mars 1967, p. 12.

+ ARCHAMBAULT, Gilles, «La joue droite de Claire Martin», Livres et Auteurs canadiens, 1966, p. 25-26.

+ ASSELIN, Normand, «Claire Martin nous parle», Incidences, janvier 1966, p. 25-29.

+ BASILE, Jean [né Bezroudnoff], «Autour de Un gant de fer, tome premier de la biographie précoce de Claire Martin. Pardon et souvenirs. Une entrevue de Jean Basile», Le Devoir, 11 décembre 1965, p. 11.

+ BELLEMARE, Yvon, «L'enfance, une histoire», Québec français, n 63, octobre 1986, p. 25-28.

+ BERNARD, Michel, [«La joue droite»], L'École, n 15, 17 mai 1969, p. 806. 
+ BERTRAND, André, «Claire Martin: la famille et le couvent», Le Quartier latin, 27 janvier 1966, p. 8.

+ — , «De Mauriac à Claire Martin. Dans un gant de fer et La joue droite», Le Quartier latin, 29 septembre 1966, p. 6.

+ BLOIS [sic], "Quelques propos de Claire Martin. Les souvenirs d'enfance ne sont pas tous affreux», Le Petit Journal, 19 décembre 1965, p. 32.

+ BOSCO, Monique, «Monique Bosco a lu Claire Martin. Née d'un tigre et d'une colombe», Le Magazine Maclean, mars 1966, p. 46.

+ — , «La rentrée littéraire [...] Trois livres décevants», Le Magazine Maclean, novembre 1966, p. 78-79.

+ BOUCHER, Claude, «La résignation fait des jeux de salauds. Claire Martin, tome deux: La joue droite», Le Sainte-Marie (Les Carnets), 3 octobre 1966, p. 8.

+ BOURBONNAIS, Nicole, "Claire Martin», William Toye (dir.), The Oxford Companion to Canadian Literature, Toronto/Oxford/New York, Oxford University Press, 1983, p. 520-521.

+ CALLAGHAN, Barry, «Women in an Iron Glove», The Telegram, $1^{\text {er }}$ février 1969.

+ CARSON, Edwina, «Fiction. In an Iron Glove [...]», Commentator, juillet-août 1969, p. 30.

+ CHENARD, Rosaire, «Dans un gant de fer», Le Cécilien, 10 février 1967, p. 7.

+ COULON, Jacques, "C'est mon homme, hélas... La complainte des femmes du Québec», Perspectives, $\mathrm{n}^{\circ}$ 17, 23 avril 1966, p. 2.

+ COUTURE, Gilles, «Humor Bids Adieu to Impotence», The Globe and Mail, 7 décembre 1968, p. 23.

+ CRAWFORD, Linda, "Pour vivre, Claire Martin doit vivre en français», La Presse, 3 octobre 1966, p. 32.

+ DALLARD, Sylvie, "Dans un gant de fer, mémoires de Claire Martin (née Montreuil)», Maurice Lemire et al., Dictionnaire des œuvres littéraires du Québec, t. IV, 1960-1969, Montréal, Fides, 1984, p. 244-246.

+ DORION, Gilles, «Dans un gant de fer [...]»,Gaétan Gervais et Jean-Pierre Pichette (dir.), Dictionnaire des écrits de l'Ontario français, Sudbury [à paraître].

+ DUHAMEL, Louis-C., «Lancement de La joue droite de Claire Martin», La Rotonde (Le Trac), 11 octobre 1966, p. 3.

+ DUHAMEL, Roger, «Claire Martin, vipère au poing», Le Droit, 31 décembre 1965, p. 7 (repris dans Québec, mai 1966, p. 84-85).

+ + , «Claire Martin nous joue un vilain tour», Photo-Journal, semaine du 16 au 23 novembre 1966, p. 16.

+ — - «Non, le livre n'est pas mort», Photo-Journal, semaine du $1^{\mathrm{er}}$ au 8 mars 1967, p. 73.

+ DUPIRE, Jacques, «Livres», Sept-Jours, 17 septembre 1966, p. 4.

+ ÉTHIER-BLAIS, Jean, «Dans un gant de fer de Claire Martin», Le Devoir, 30 décembre 1965, p. 10.

+ — - «Livres en français. Romans», University of Toronto Quarterly, juillet 1966, p. 522-523.

+ — - «La joue droite», Le Devoir, 17 septembre 1966, p. 11 (repris dans Signets II, p. 224-228, sous le titre «Entre femmes seules»).

+ FERRON, Jacques, «Dans un gant de fer», Le Magazine Maclean, février 1971, p. 42 et 44.

+ FOURNIER, Roger, «Claire Martin: des pages écorchantes», Le Petit Journal, 16 octobre 1966, p. 51.

+ FRANCION [pseudonyme de Lisette Morin], "Claire Martin et le "livre noir" de son père», Le Progrès du Golfe, 24 décembre 1965, p. 19.

+ — - "Les noirs souvenirs d'un "vert paradis", La joue droite», Le Progrès du Golfe, 29 septembre 1966, p. 15.

+ FRÉCHETTE, Jean, «Notes de lecture. Les enfances de Claire Martin», L'Action nationale, décembre 1966, p. 386-389.

+ GAGNON, Lysiane, «Du Québec des années noires à celui de 1968», La Presse (section Magazine), 30 décembre 1967, p. 3-5.

+ GAY, Paul, «Dans un gant de fer», Le Droit, 24 décembre 1965, p. 7.

+ GOURD, Anne, «Au pays des livres. Et puis le pardon. Dans un gant de fer, par Claire Martin», Actualité, mai 1966, p. 33.

+ —— , "Au pays des livres. L'enfance terrorisée. La joue droite», Actualité, décembre 1966, p. 44-45. 
+ H[OULE], R[ené], «Les mémoires de Claire Martin: Dans un gant de fer», L'Écho de Frontenac, 28 novembre 1968, p. 19.

+ — - "Les mémoires de Claire Martin: Dans un gant de fer», Ici Radio-Canada, 30 novembre 1968, p. 11.

+ KUNSTLER Marion, "Two Roads Lead to Success», The Gazette, 19 octobre 1967, p. 13.

+ LAPOINTE, Pierre, «Nos romans noirs», Le Bien public, 27 janvier 1967, p. 1 (repris dans L'Action, $1^{\text {er }}$ février 1967, p. 4).

+ LÉGARÉ, Céline, «Claire Martin ou le procès de l'éducation féminine», La Patrie, 18 septembre 1966, p. 20.

+ LÉGARÉ, Romain, «Chronique. [...] Prix littéraires», Culture, juin 1966, p. 196.

$+\ldots$, «Les livres canadiens. Dans un gant de fer», Culture, juin 1966, p. 210-211.

+ + - «Les livres canadiens. La joue droite», Culture, décembre 1966, p. 484-486.

+ LOCKQUELL, Clément, «Un livre de Claire Martin. Dans un gant de fer», Le Soleil, 8 janvier 1966, p. 6.

+ —_ «La joue droite de Claire Martin», Le Soleil, 17 septembre 1966, p. 8 (repris dans Québec, février 1967, p. 100-102).

+ MAJOR, André, «Le [sic] gant de fer», Le Petit Journal, semaine du 16 janvier 1966, p. 36.

+ — - «Dans un gant de fer. Claire Martin et son univers», Vient de paraître, novembre 1966, p. 28-29.

+ MARCOTTE, Gilles, "Claire Martin, le "vieux démon ironique" et les surprises de la mémoire», La Presse, 11 décembre 1965, p. 5.

+ — - "Dans un gant de fer ou l'histoire d'un combat», La Presse (supplément), 24 décembre 1965, p. 4 (repris dans Les bonnes rencontres, chroniques littéraires, p. 161-165, sous le titre «Le grand combat de Claire Martin»).

+ MARTEL, Réginald, «Le malheur tue rarement», La Presse, 6 février 2000, p. B-4.

+ MORIN, Yvon, «Livres à lire au coin du feu. La joue droite», L'Évangéline, 14 janvier 1967, p. 4.

+ NORMANDEAU, Mimi, «Dans un gant de fer - Claire Martin», Bulletin. Société d'étude et de conférences, juin 1966, p. 59.

+ OUELLET, Lise, "Du monde raconté à l'autoportrait: Dans un gant de fer [...]», Revue de l'Université de Moncton, 1986, p. 167-186.

+ PALLASCIO-MORIN, Ernest, «La joue droite de Claire Martin», L'Action, 4 novembre 1966, p. 21.

+ PARADIS, Suzanne, Femme fictive, femme réelle. Le personnage féminin dans le roman féminin canadienfrançais, 1884-1966, Québec, Garneau, 1966, p. 149-159.

+ PELLETIER-BAILlARGEON, Hélène, «Les bonnes sœurs de Claire Martin», Maintenant, février 1967, p. 64-65.

+ P[ILON], J[ean]-G[uy], "Les écrits. Dans un gant de fer», Liberté, nº 43, janvier-février 1966, p. 68-69.

+ —_, «Notes de lecture. La joue droite, par Claire Martin», Liberté, n ${ }^{\text {os }}$ 47-48, septembre-décembre 1966, p. 178.

+ PILOTTE, Hélène, «La romancière Claire Martin. Analyste de l'amour et de la femme. Un reportage de Hélène Pilotte», Châtelaine, juin 1965, p. 28-29, p. 46-49 (avec un extrait de Dans un gant de fer, p. 30).

+ POISSANT, Georges, «Le livre du mois», Le Sainte-Marie (Les Carnets), février 1966, p. 4.

+ P[ONTAUT], A[lain], «Claire Martin et l'exorcisme d'une adolescence», La Presse (supplément), 10 septembre 1966, p. 5.

+ — - «L'enfant devant les monstres», La Presse (supplément), 17 septembre 1966, p. 4.

+ POULIOT, Conrad, "Vous avez le dernier mot. Une affaire Claire Martin», Châtelaine, "Courrier des lecteurs», avril 1966, p. 148.

+ POUPART, Jean-Marie, «Claire Martin: “Oui, j’aime mon père!”», L'Illettré, avril-mai 1971, p. 4.

+ RANCOURT, Marcel, «Claire Martin», Campus estrien (numéro spécial), avril 1968, p. 9.

+ RENAUD, André, "Lecture du mois. Dans un gant de fer», Relations, avril 1966, p. 116.

+ - - ,La joue droite», Relations, novembre 1966, p. 314.

+ RIVARD, Yvon, «Claire Martin. Notre théoricienne du cœur humain», L'Action nationale, juin 1967, p. 10411046. 
+ ROBIDOUX, Réjean, «Livres en français. Romans et nouvelles», University of Toronto Quarterly, juillet 1968, p. 571-572.

+ ROY, Claire, «Dans un gant de fer. Claire Martin dévoile la dure étreinte de son enfance», Le Nouvelliste, 12 février 1966, p. 5.

+ S[AIN]T-GERMAIN, André, "Cris-tics», Le Carabin, 27 septembre 1966, p. 11.

+ SAINT-MARTIN, Fernande, «Éditorial. On ne peut prêter aux autres ses souvenirs d'enfance», Châtelaine, février 1966, p. 1.

+ SAINT-ONGE, Paule, "Châtelaine a lu pour vous. Deux mondes fantastiques», Châtelaine, février 1966, p. 44.

+ — , "Châtelaine a lu pour vous. Les Mémoires de Claire Martin [...]», Châtelaine, novembre 1966, p. 17.

+ SAMSON, Jean-Noël, «Dialogue avec les livres d'hier et d'aujourd'hui. Dans un gant de fer de C. Martin», Lectures, avril 1966, p. 199-200.

+ STAFFORD, Jan, "Claire Martin: “J'ai tout pardonné"», Désormais, avril 1966, p. 15.

+ STARRS, Roy, «Inside View of Woman in a Straitjacket Society», The Province (Supplement), 21 février 1969, p. 5, Vancouver.

+ STRATFORD, Philip, "Quebec Books», The Globe Magazine, 29 octobre 1966, p. 26.

+ TARD, Louis-Martin, «Claire Martin, un rayon de soleil après l'orage», La Patrie, 7 août 1966, p. 45

+ THÉBERGE, Jean-Yves, «En retard. Dans un gant de fer», Le Canada français, 25 août 1966, p. 22.

+ _ _ «'il n'y avait pas le style...», Le Canada français, 29 septembre 1966, p. 42.

+ THÉRIAULT, Jacques, «Dix minutes avec Claire Martin. C'était une très bonne histoire à raconter», Le Devoir, 10 septembre 1966, p. 14.

+ THÉRIO, Adrien [né Thériault], «Et voici le père. Dans un gant de fer de Claire Martin», Livres et Auteurs canadiens, 1965, p. 49-51.

+ TRANQUILLE, Henri, «Culture et loisirs. Livres. La joue droite», Sept-Jours, 1 1er octobre 1966, p. 47.

+ VALIQUETTE, Bernard, «Ā la page. Le livre de la semaine. Dans un gant de fer», Échos-Vedettes, 25 décembre 1965, p. 24.

+ — , «Ā la page. La joue droite de Claire Martin», Échos-Vedettes, 15 octobre 1966, p. 22.

+ WARWICK, Jack, «Translating Brilliance», Canadian Literature, automne 1969, p. 82-84.

\section{II.5. Les morts}

+ [ANONYME], «Claire Martin. Les morts», L'Action-Québec, 16 janvier 1971, p. 18.

+ _ _ "Claire Martin écrivain», Ici Radio-Canada, 10 juillet 1971, p. 9.

+ — , «Canadiana [...] Les morts», L'Action-Québec, 18 septembre 1971, p. 18.

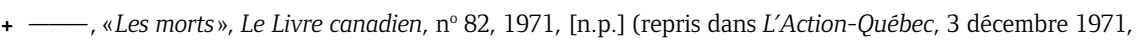
p. 16 et dans L'Église canadienne, décembre 1972, p. 307-308)

+ ARTHUR, Sheilah, «Two More Excellent Made-in-Quebec Novels», The Gazette, 23 janvier 1971, p. 42.

+ CHARLAND, Jean-Paul, "Chronique littéraire. Les morts», Le Médecin du Québec, mars 1971, p. 63.

+ DALLARD, Sylvie, «Les morts, roman de Claire Martin», Maurice Lemire et al., Dictionnaire des œuvres littéraires du Québec, t. V, 1970-1975, Montréal, Fides, 1987, p. 569-571.

+ DUHAMEL, Roger, «Une passion d'hier et une passion d'aujourd'hui», Le Droit, 2 janvier 1971, p. 7.

+ ÉTHIER-BLAIS, Jean, «Claire Martin: l'analyse de l'Amour», Le Devoir, 19 décembre 1970, p. 11.

+ HARE, John, «Documentation», Bulletin du Centre de recherche en civilisation canadienne-française, avril 1972, p. 25-26.

+ L'ILLETTRÉ [pseudonyme de Harry BERNARD], «Roman de Claire Martin qui s'adapterait vite au thêâtre», Le Bien public, 26 février 1971, p. 2.

+ KUSHNER, Éva, «Ā propos des Morts de Claire Martin», Livres et Auteurs québécois, 1970, p. 32-36.

+ LACROIX, Georgette, "Arts et littérature», L'Action, 6 février 1971, p. 18.

+ LAMOUREUX, Georgette, «Nouvelles brèves», Le Droit, 24 octobre 1970, p. 7. 
+ MARTEL, Réginald, «Écrire: entre le + et le -», La Presse, 16 janvier 1971, p. D-3.

+ RENAUD, André, «Un roman de Claire Martin. Les morts», Le Droit, 26 décembre 1970, p. 6.

+ — , «Claire Martin et les mots (2)», Le Droit, 2 janvier 1971, p. 6.

+ ROBIDOUX, Réjean, «Romans, récits, nouvelles, contes», University of Toronto Quarterly, été 1971, p. 431.

+ SAINT-ONGE, Paule, "Les morts», Châtelaine, février 1971, p. 44.

+ T[HÉBERGE], J[ean]-Y[ves], «Un grand amour ou une grande chipure», Le Canada français, 27 janvier 1971, p. 34.

+ TREMBLAY, Denis, «Deux lancements, un jour», Montréal-Matin, 11 décembre 1980, p. 19.

\section{II.6. "Moi, je n'étais qu'espoir"}

+ [ANONYME], «Au TRV, une œuvre adaptée de Claire Martin», Le Droit, 9 mars 1972, p. 26.

+ _ _ «Au Rideau Vert. Adaptation d'un roman de Claire Martin», La Presse, 10 mars 1972, p. C-4.

+ — , «Pièce de Claire Martin au Rideau Vert», La Tribune, 14 mars 1972, p. 9.

+ BEAULIEU, Michel, «Au Rideau-Vert, un spectacle prétentieux et... inutile», Le Devoir, 25 mars 1972, p. 17.

+ BROUSSEAU, Jean-Paul, «Un peu plus loin... et voilà Claire Martin dramaturge», La Presse, 18 mars 1971, p. D-4.

+ DASSYLVA, Martial, «En attendant la deuxième chance», La Presse, 22 mars 1972, p. F-13.

+ D’AUTEUIL, Georges-Henri, «Au Rideau Vert: Moi, je n'étais qu'espoir», Relations, mai 1972, p. 156.

+ HÉBERT, François, «Moi, je n'étais qu'espoir de Claire Martin», Livres et Auteurs québécois, 1972, p. 125-126.

+ H[OULE], R[ené], «Sur toutes les scènes du monde. Les morts», Ici Radio-Canada/Radio, 3 février 1973, p. 19.

+ JASMIN, Claude, «Arts et spectacles [...] L'aveugle fait place à l'espoir?», Le Journal de Montréal, 9 mars 1972, p. 23

+ — , "Arts et spectacles. [...] Au Rideau Vert: Les morts de Claire Martin», Le Journal de Montréal, 21 mars 1972, p. 19.

+ LAMY, Suzanne, «Une œuvre où la femme est libre. Pour Claire Martin un nouveau départ: la création dramatique», Châtelaine, mars 1972, p. 12.

+ MAHEUX-FORCIER, Louise, "Claire Martin: Moi, je n'êtais qu'espoir», Archives des lettres canadiennes, t. V, Le théâtre canadien-français. Évolution-Témoignages-Bibliographie, Montréal, Fides, 1976, p. 619-622.

+ SUISSA, Danièle J., "Moi, je n'étais qu'espoir"», Thêâtre du Rideau Vert, saison 71-72, 17 mars 1972, [n.p.].

+ TISSEYRE, Pierre, «Moi, je n'étais qu'espoir», Théâtre du Rideau Vert, saison 71-72, 17 mars 1972, [n.p.].

+ VICQ, Victor, «Victor Vicq a vu et entendu [...] Le roman Les morts à la scène», Le Droit, 21 février 1972, p. 5.

+ _ , «Victor Vicq a vu et entendu [...] Précisions de Claire Martin», Le Droit, 25 février 1972, p. 5.

\section{II.7. La petite fille lit}

+ DORION, Gilles, «La petite fille lit», Gaétan Gervais et Jean-Pierre Pichette (dir.), Dictionnaire des écrits de l'Ontario français, Sudbury [à paraître].

\section{II.8. Le choix de Claire Martin dans l'œuvre de Claire Martin}

+ DUHAMEL, Roger, «Point de vue. À propos de Claire Martin», La Presse, 21 décembre 1984, p. A-6.

+ DUVAL, Monique, "Claire Martin choisit quelques pages de son œuvre littéraire», Le Soleil, 7 décembre 1984, p. C-4.

+ LAFRENIÈRE, Suzanne, «Le choix de Claire Martin dans l'œuvre de Claire Martin», Le Droit, 25 mai 1985, p. 38.

+ MARTEL, Réginald, «Littérature. Trois brochures sur l'UNEQ [...] Cinq lancements [...]», La Presse, 26 novembre 1984, p. B-8.

+ ROYER, Jean, «Le choix de Claire Martin», Le Devoir, 8 décembre 1984, p. 29.

+ THÉRIO, Adrien, «Lectures. Le choix de Claire Martin dans l'œuvre de Claire Martin [...], Lettres québécoises, $\mathrm{n}^{\circ} 37$, printemps 1985, p. 73. 


\section{II.9. Québec, des écrivains dans la ville [coord. Gilles Pellerin]}

+ LACHANCE, Micheline, «Michel Chartrand, grandeur nature. [...] Le Québec de Claire Martin», Actualité, $1^{\text {er }}$ mai 1995, p. 117.

\section{II.10. Toute la vie}

+ [ANONYME], «Arts et Spectacles», Impact Campus, 16 mars 1999, p. 27.

+ ——, «À la Dictée», Le Soleil, 19 mars 1999, p. A-4.

+ — , «Toute la vie», Châtelaine, avril 1999, p. 26.

+ AUDET, René, «Claire Martin. Toute la vie», Québec français, n 115, automne 1999, p. 10.

+ BARBANCHE, Maryse, «Pour non-liseurs. Amours et oignons verts», Liberté, no 246, décembre 1999, p. 113.

+ CAOUETTE, Marie, «Claire Martin écrivaine. À 85 ans, sereine et active», Le Soleil, 8 mars 1999, p. A-1 et A-2.

+ — , «Entrevue avec l'écrivaine Claire Martin. "J'aimerais pouvoir inventer un secret"», Le Soleil, 8 mars 1999, p. B-11.

+ CHAREST, Rémy, «Claire Martin. Écrire sans attaches», Le Devoir, $20-21$ mars 1999, p. D-1 et D-2.

+ CHARTRAND, Robert, «Nature heureuse et tête bien faite», Le Devoir, 13-14 mars 1999, p. D-3.

+ _ _ «Fin de saison. Un coup d'œil partiel et partial sur ce qui s'est écrit, fait et dit», Le Devoir, 12-13 juin 1999, p. D-4.

+ DROUIN, Serge, «Toute la vie. Nouvelles et souvenirs de Claire Martin», Le Journal de Québec, 15 mars 1999, p. 50.

+ JOUBERT, Lucie, «Trois générations. [...] Vieux rose», Spirale, nº 169, novembre-décembre 1999, p. 26.

+ LACROIX, Georgette, «La Vitrine à Jojo», Reflets [journal de l'Association québécoise des retraités des secteurs public et parapublic], juin 1999, p. 8.

+ LAPLANTE, Laurent, «Toute la vie», Nuit blanche, nº 76, automne 1999, p. 7.

+ LE BEAU, Hélène, "Elle a envie de lire. Claire Martin. Toute la vie», Elle Québec, n 117, mai 1999, p. 34.

+ LORD, Michel, «Les grands "anciens" persistent et signent», Lettres québécoises, n 96, hiver 1999, p. 32.

+ MALAVOY-RACINE, Tristan, "Claire Martin. Toute la vie. Le chant retrouvé», Voir (Québec), 11-17 mars 1999, p. 22 (reproduit dans Voir (Montréal), $1^{\text {er }}$ avril 1999).

+ MARCOTTE, Gilles, «Les livres. Lire, écrire, pourquoi donc?», L'Actualité, juillet 1999, p. 97.

+ MARCOUX, Stephen, «Une nouvelle vie qui commence. Claire Martin, écrivaine de la Haute-Ville. Retrouver l'amour de l'écriture à 85 ans», Carrefour, 6 juin 1999, p. 17.

+ MARTEL, Réginald, «Les bonheurs de Claire Martin», La Presse, 14 mars 1999, B-1.

+ PAYER, Carole, «Lecture [...] Toute la vie», Le Journal de Québec, 10 avril 1999, p. 35.

+ [PÉAN], Stanley, «Claire Martin: le retour», Le Libraire, n 3, mars 1999, p. 1.

+ STANTON, Julie, «Claire Martin relève le gant», Madame au foyer, mai 1999, p. 44-45.

\section{II.11. L'amour impuni}

+ [ANONYME], «L'amour impuni, l'auteure récompensée», Le Soleil, 13 avril 2000, p. A-1.

+ — , «Lecture. L'amour impuni», Le Journal de Québec, 29 avril 2000, p. 35.

+ _ , «L'amour impuni, par Claire Martin, L'instant même», Magazine Le Clap, n 86, avril-juin 2000, p. 22.

+ BIRON, Michel, «Devant quelques photos de famille», Voix et Images, n 76, automne 2000, p. 183.

+ BOIVIN, Aurêlien, «Claire Martin. L'amour impuni», Québec français, n 118, été 2000, p. 21.

+ BORDELEAU, Francine, "Claire Martin. L'amour impuni», La Gazette des femmes, septembre-octobre 2000, p. 42.

+ CHARTRAND, Robert, «Et pourquoi pas le bonheur?», Le Devoir, 8-9 avril 2000, p. D-1 et D-2.

+ CLÉMENT-MORIN, Geneviève, «Le libraire craque», Le Libraire, été 2000, p. 11.

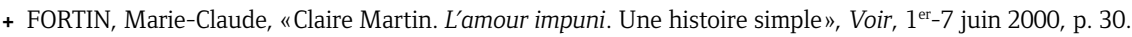

+ KARCH, Pierre, «Roman. Letters in Canada/Lettres canadiennes 2000», University of Toronto Quarterly, hiver 2001-2002, p. 412. 
+ LAROUCHE, Réjeanne, «L'amour impuni», Nuit blanche, [2000], [Internet].

+ LAURIN, Danielle, «Elle a envie de lire. Claire Martin. L'amour impuni», Elle Québec, no 130, juin 2000, p. 40.

+ LEPAGE, Jocelyne, «Invités prestigieux et débats au Salon du livre de Québec», La Presse, 12 avril 2000, p. E-3.

+ MARCOTTE, Gilles, «Les liaisons de l'une, les pièces de l'autre. Claire Martin traite sur le ton intimiste les amours entre deux hommes [...]», L'Actualité, $1^{\text {er }}$ septembre 2000, p. 67.

+ MARTEL, Réginald, «Ils vécurent heureux et n'eurent pas beaucoup d'enfants», La Presse, 16 avril 2000, p. B-5.

+ MOREAULT, Éric, «L'amour récompensé. Claire Martin obtient la médaille de l'Académie des lettres», Le Soleil, 13 avril 2000, p. C-3.

+ PÉAN, Stanley, «Une nouvelle bibliothèque rose?», Le Libraire, été 2000, p. 8.

+ ROCHON, Claude, «Il y a des amours heureux!», Zone, mai 2000, p. 37.

+ SOULIÉ, Jean-Paul, «Sur la scène de l'actualité/Semaine du 16 avril 2000. Claire Martin», La Presse, «La personnalité de la semaine», 16 avril 2000, p. A-16.

\section{12 . La brigande}

+ BOIVIN, Aurélien, «La brigande», Québec français, n 125, printemps 2002, p. 23.

+ BROCHU, André, «La brigande, le mourant et le joueur de flûte», Lettres québécoises, n 106, été 2002, p. 13.

+ CHARTRAND, Robert, «Le temps d'un récit», Le Devoir, 24-25 novembre 2001, p. D-3.

+ LAROUCHE, Réjeanne, "Claire Martin. La brigande [...]", Nuit blanche, nº 87, été 2002, p. 37.

+ MARCOTTE, Gilles, «De secrets en énigmes. Claire Martin, Antonine Maillet et Nicole Brossard livrent des "histoires à tiroirs" où l'homme joue un rôle... décoratif", L'Actualité, 15 mars 2002, p. 91.

+ MARTEL, Réginald, «Du talent pour le bonheur», La Presse, 9 décembre 2001, p. B-4.

+ SYLVESTRE, Paul-François, «Trois romans québécois, dont le plus attendu reste décevant», L'Express, 22-28 janvier 2002, p. 9.

\section{II.13. Il s'appelait Thomas}

+ BOIVIN, Aurélien, «Il s'appelait Thomas», Québec français, n 130, été 2003, p. 15-16.

+ MARTEL, Réginald, «Harlequin visité par le style», La Presse, 20 avril 2003, p. F-2.

+ POULIOT, Sophie, «Les plaisirs démodés», Le Devoir, 12-13 avril 2003, p. F-5.

+ TRUDEL, Sylvain, «Il s'appelait Thomas, de Claire Martin. Un roman qui meurt trop tard», Le Soleil, 13 avril 2003, p. B-5.

\section{OU VR AGES GÉNÉRAUX}

+ ARGUIN, Maurice, Le roman québécois de 1944 à 1965. Symptômes du colonialisme et signes de libération, Québec, Université Laval, Centre de recherche en littérature québécoise, 1985, p. 140, 152, 161, 189; Montréal, l'Hexagone, 1989, p. 182, 197, 209, 247.

+ BESSETTE, Gérard, Lucien GESLIN et Charles PARENT, Histoire de la littérature canadienne-française, Montréal, Centre éducatif et culturel, 1968, p. 587-593.

+ DUHAMEL, Roger, Manuel de littérature canadienne-française, Montréal, Éditions du Renouveau pédagogique, 1967, p. 136-137.

+ GAY, Paul, Notre littérature, Montréal, HMH, 1969, p. 168-169.

+ - Notre roman, Montréal, Hurtubise HMH, 1973, p. 82, 127-128, 145, 147-148, 167.

+ GRANDPRÉ, Pierre de, Histoire de la littérature française du Québec, t. IV, Montréal, Beauchemin, 1969, p. 88-94.

+ HAMEL, Réginald, John HARE et Paul WYCZINSKY, Dictionnaire pratique des auteurs québécois, Montréal, Fides, 1976, p. 484-485. 
+ — Dictionnaire des auteurs de langue française en Amérique du Nord, Montréal, Fides, 1989, p. 952-954.

+ HAMEL, Réginald (dir.), Panorama de la littérature québécoise contemporaine, Montréal, Guérin, 1997, p. 314, 317, 321, 354, 355.

+ MAILHOT, Laurent, La littérature québécoise, Paris, Presses universitaires de France, coll. «Que sais-je?» $\mathrm{n}^{\circ} 1579,1974$, p. 119.

+ ——, La littérature québécoise depuis ses origines, Montréal, Typo, 1997, p. 159, 174, 337.

+ RACINE, Claude, L'anticléricalisme dans le roman québécois 1940-1965, Montréal, Hurtubise HMH, coll. «Littérature. Les Cahiers du Québec», nº 6, 1972, p. 36, 118.

+ VIGNEAULT, Robert, Claire Martin, son œuvre, les réactions de la critique, préface de Roger Le Moine, Montréal, CLF Pierre Tisseyre, 1975, 215 p.

\section{ARTICLES GÉNÉRAUX SUR CLAIRE MARTIN ET SON EUVRE}

+ [ANONYME], «Wilfrid Lemoine anime “Gros Plan"; sa 1 ère invitée: Claire Martin», Ici Radio-Canada, 30 mai 1970, p. 5.

+ ——, «Sondage au Québec. Diverses personnalités féminines répondent à nos questions. [...] Claire Martin. Romancière [...]», Châtelaine, novembre 1970, p. 26-28, 56.

+ _- "Qui est vraiment Claire Martin», Ici Radio-Canada, janvier 1971, p. 16.

+ BEAULIEU, Alain, «Claire Martin», Entretien. Enregistrement sonore, Québec, Alain Beaulieu, coll. «Je vous entends écrire», 2002, 1 disque, CKRL-MF 89,1.

+ BOISCLAIR, Isabelle, «Claire Martin, tous genres confondus», Québec Studies, n² 26, automne 1998/hiver 1999, p. 53.

+ BOURBONNAIS, Nicole, "Robert Vigneault à l'écoute de Claire Martin», Lettres québécoises, n 3, septembre 1976, p. 27-29.

+ BOURNEUF, Roland, «Claire Martin, la note juste», Nuit blanche, nº 91, été 2003, p. 23-27 [précédé d'une photo de Claire Martin, p. 22].

+ BRUNELLE, Christiane, "Au Salon du Livre. "Mes personnages féminins sont égaux aux hommes" (Claire Martin)», Le Soleil, 27 octobre 1964, p. 9.

+ CHANTAL, Geneviève de, "Une heure avec Claire Martin», Le Droit, 3 juin 1961, p. 11.

+ CLOUTIER, Cécile, "Panorama de la littérature québécoise d'aujourd'hui», The Canadian Modern Language Review, octobre 1974, p. 8-32 (voir p. 9).

+ DÉPARTEMENT DES LITTÉRATURES, UNIVERSITÉ LAVAL, «Rencontre de Claire Martin, le 11 octobre 1972, à la Faculté des lettres de l’Université Laval», Québec, novembre 1972, 22 pages [tapuscrit].

+ DORION, Gilles, «La littérature québécoise contemporaine 1960-1977. Le roman», Études françaises, «Petit manuel de littérature québécoise», nos 3-4, octobre 1977, p. 314-316.

+ ——, «Martin, Claire», L'encyclopédie du Canada, t. 2, Montréal, Stanké, 1987, p. 1200.

+ — , «Martin, Claire», The Canadian Encyclopedia, Edmonton, Hurtig Publishers, rééd. 1988, p. 1310.

+ FAVREAU, Mariane, "Les héroïnes livresques de Claire Martin face à la "critique" de Suzanne Paradis», $L a$ Presse, 8 mars 1972, p. D-1.

+ GIRARD, Marie-Claire, «L'entrevue. Claire Martin. Dans un gant de velours», Le Devoir, $1^{\text {er }}$ avril 1997, p. B-1.

+ GODIN, Gérald, «Notre littérature, image de notre milieu? ou l'art de discuter de faux problèmes», Le Nouvelliste, 8 octobre 1960, p. 10.

+ IQBAL, Françoise et Gilles DORION, «Claire Martin. Une interview», Canadian Literature, n 82, automne 1979, p. 59-77.

+ KAYE, Françoise, "Claire Martin ou le "je" aboli», Incidences, mai-décembre 1980, p. 49-58.

+ KOSKI, Raija, "Claire Martin: la femme et l'écriture», Archives des lettres canadiennes, t. VIII, Le roman contemporain au Québec (1960-1985), Montréal, Fides, 1992, p. 349-362. 
+ LAMOUREUX, Georgette, «Entretien avec Claire Martin», Le Droit, 14 octobre 1972, p. 13.

+ LEGAULT, Agathe, "Claire Martin, sa sagesse, ses jugements, sa géographie du monde», Bulletin de la Société des écrivains canadiens, été 1970, p. 8-12.

+ LEGRIS, Renée, «Hommage à Claire Martin», Arcade, n 40, «Voix internationales», $2^{\mathrm{e}}$ trimestre 1997, p. 107117.

+ LÉVESQUE, Gaëtan, «Entretien. Rompre le silence», XYZ. La revue de la nouvelle, printemps 1985, p. 5-10.

+ RICARD, François, «Claire Martin retrouvée», Les livres d'ici, n 48, 1975, [n.p.]

+ ROBERT, Lucette, «Ce dont on parle», La Revue populaire, juillet 1945, p. 12.

+ ROBERTS, Katherine A., «Genres intimes et discours politique: Claire Martin, Andrée Maillet et Francine Noël», Lucie Joubert (dir.), Trajectoires au féminin dans la littérature québécoise (1960-1990), Québec, Nota bene, 2000, p. 49-66.

+ ROBIDOUX, Réjean, «Claire Martin, romancière», Études françaises, n 2, juin 1965, p. 67-86.

+ — - «Robert Vigneault, Claire Martin, son œuvre, les réactions de la critique», Livres et Auteurs québécois, 1976 , p. 249-250.

+ ROBIDOUX, Réjean et André RENAUD, Le roman canadien-français du vingtième siècle, Ottawa, Éditions de l'Université d'Ottawa, 1966, 214 p.

+ ROBILlARD, Jean-Paul, «Chez Claire Martin. Ottawa ne l'a pas “eue" », Le Petit Journal, semaine du 4 juin 1961, p. B-30.

+ ROY, Monique, «Un écrivain se raconte», Châtelaine, juin 2000, p. 68.

+ SARRAZIN, Pierre, «Évocation d'écrivains de chez nous: Claire Martin et Monique Champagne», Ici RadioCanada, 28 juin 1969, p. 9.

+ SENÉCAL, Louise-Marie, "Claire Martin ou le refus du rôle traditionnel de mère», Le Devoir, 19 décembre 1964, p. 13-14.

+ TARDIF, Jacqueline, «Bio-bibliographie de Claire Martin», Université de Montréal, École de bibliothéconomie, 1970, $14 \mathrm{f}$.

+ TASSÉ, Emmanuelle, «Avoir 20 ans au XXe siècle?», Femmes Plus, décembre 1999, p. 33.

+ TASSO, Lily, «"Une femme libérée est plus intéressante" -Claire Martin», La Presse, 15 décembre 1970, p. C-4.

+ URBAS, Jeannette, «Le jeu et la guerre dans l'œuvre de Claire Martin», Voix et images du pays VIII, 1974, p. 133-147.

+ WYCZINSKI, Paul, "Panorama du roman canadien-français», Archives des lettres canadiennes, t. III, Le roman canadien-français. Évolution-Témoignages-Bibliographie, Montréal, Fides, 1964, p. 11-35 (voir p. 21, 25-26).

+ YOKEN, Mel B., Entretiens québécois, vol. I, Montréal, CLF Pierre Tisseyre, 1986, 187 p. (voir «Claire Martin», p. [115]-126).

\section{MÉMOIRES ET THËSE SUR L'EUVRE DE CLAIRE MARTIN}

+ ABRIOUX, Ann Lewese, «Love in the Works of Claire Martin», mémoire de maîtrise, Hamilton, McMaster University, 1973, iv-65 f.

+ BOILY, Johanne, «Le féminisme dans l'œuvre de Claire Martin», mémoire de maîtrise, Winnipeg, University of Manitoba, 1985, ii-88 f.

+ COSTISELLA, Béatrice, «Le portrait de son père par Claire Martin dans son autobiographie intitulée Dans un gant de fer et La joue droite», mémoire de maîtrise, Dijon, Université de Bourgogne, 147 f.

+ KOSKI, Raija Hellevi, "L'échec de l'amour dans Quand j'aurai payé ton visage de Claire Martin», mémoire de maitrise, London, University of Western Ontario, 1977, v-96 f.

+ — - «La femme et l'espace chez Claire Martin», thèse de doctorat, Toronto, University of Toronto, 1985, $255 \mathrm{f}$. 
+ MARSOLAIS, Jeanne Georgette, «Naissance et mort de l'amour dans les romans et nouvelles de Claire Martin", mémoire de maîtrise, Montréal, McGill University, 1972, 150 f.

+ MOREY, Elizabeth M., "Claire Martin à travers son œuvre», mémoire de maîtrise (Romance Studies), Calgary, University of Calgary, 1973, vi-93 f.

+ SMITH, Diane Lee, "Claire Martin in English: Theory and Practice of Literary Translation», mémoire de maîtrise, Edmonton, University of Alberta, 1976, $115 \mathrm{f}$.

+ STERNS, Margaret M[elinda], «Le couvent dans l'œuvre de Laure Conan, Claire Martin et Anne Hébert», mémoire de maîtrise, Kingston, Queen's University, 1986, 127 f.

+ THIBAUDEAU, Huguette, «Les femmes dans l'œuvre de Claire Martin», mémoire de maîtrise, Vancouver, University of British Columbia, 1975, iv-119 f.

+ TREMBLAY, Rose-Marie, «Le personnage-écrivain dans Les morts de Claire Martin», mémoire de maîtrise, Vancouver, University of British Columbia, 1987, $125 \mathrm{f}$.

\section{PRIX ET DISTINCTIONS}

+ Prix du Cercle du livre de France (1958) pour Avec ou sans amour.

+ Prix de la Province de Québec et Prix France-Québec (1966) pour Dans un gant de fer.

+ Prix du Gouverneur général du Canada (1967) pour La joue droite.

+ Membre de la Société royale du Canada (1967).

+ Médaille de la Reine Elizabeth II (1977).

+ Officier de l'Ordre du Canada (1984).

+ Médaille de l'Académie des Lettres du Québec (2000).

+ Compagnon de l'Ordre du Canada (2002).

+ Médaille de la Reine Elizabeth II [jubilé] (2002). 